\title{
MULTI-WAVELENGTH PREDICTIONS OF THE DIM
}

\author{
M. R. Schreiber, ${ }^{1}$ J.-M. Hameury, ${ }^{1}$ and J.-P. Lasota ${ }^{2}$
}

Using the disc instability model (DIM) and a simple but reasonable model for the X-ray, extreme UV, UV and optical emission we investigate the multi-wavelength properties of dwarf novae. We discuss the predictions of the model in the context of the observationally best studied systems, i.e. SS Cyg and VW Hyi. We use the version of the DIM described in Buat-Ménard et al. (2001). The local spectrum of the emission from the disc is assumed to be given by Kurucz (1993,VizieR On-line Data Catalog). We also take into account emission from the (irradiated) secondary, the white dwarf, the hot spot, and the boundary layer. The latter is expected to be the dominan: source of EUV and hard X-ray photons. We assume that the boundary layer is optically thin (X-ray emitting) if the mass accretion rate is below $\dot{M}_{\mathrm{cr}}=10^{16} \mathrm{gs}^{-1}$ and optically thick (radiating at EUV wavelengths) otherwise. The UV and EUV delays predicted for SS Cyg are somewhat longer for inside-out than for outside-in outbursts but in agreement with the observations (Fig. 1). Increased X-ray emission at the beginning and end of the outbursts, comparable to the observed ones, is a natural outcome of the model. X-ray observations during quiescence strongly support the idea of truncation. Fig. 2 shows light curves predicted for VW Hyi by the Enhanced Mass Transfer (EMT) model. It is assumed that irradiation of the secondary is causing enhanced mass transfer during outburst. As observed the precursor outbursts predicted by the EMT are more pronounced at short wavelengths. In contrast to the competing thermal tidal instability (TTI) model, the predictions of the EMT are very sensitive to variations of the mass transfer rate. Concerning the large variety of observed phenomena in SUUMa systems the EMT is the more promising scenario. For more details see Schreiber et al. (2003b).

\section{REFERENCES}

Buat-Ménard, V., et al., 2001, A\&A, 366, 612

Schreiber, M. R., et al., 2003, A\&A, 410, 239

\footnotetext{
${ }^{1}$ Obs. de Strasbourg, 11 rue de l'Lniversité, F-67000 Strasbourg.

${ }^{2}$ Inst. d'Astrophys. de Paris, 98bis Boul. Arago, F-75014 Paris.
}
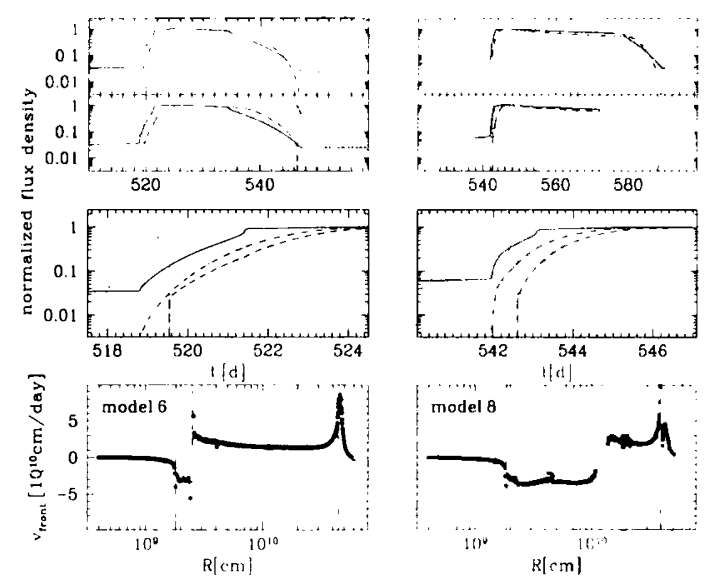

Fig. 1. Predicted normalized optical (solid line), LV flux (dashed-dotted), X-ray (dotted), EUV (long dashed) emission, and the heating front velocity during the rise (bottom) for SSCyg. The inner disc is assumed to be truncated during quiescence. Model 6 is of the inside out type whereas in model 8 the heating front starts far from the white dwarf. The vertical lines in the bottom panels indicate the position of the outer and inner edge of the disc during quiescence as well as the radius at which the heating front was triggered.

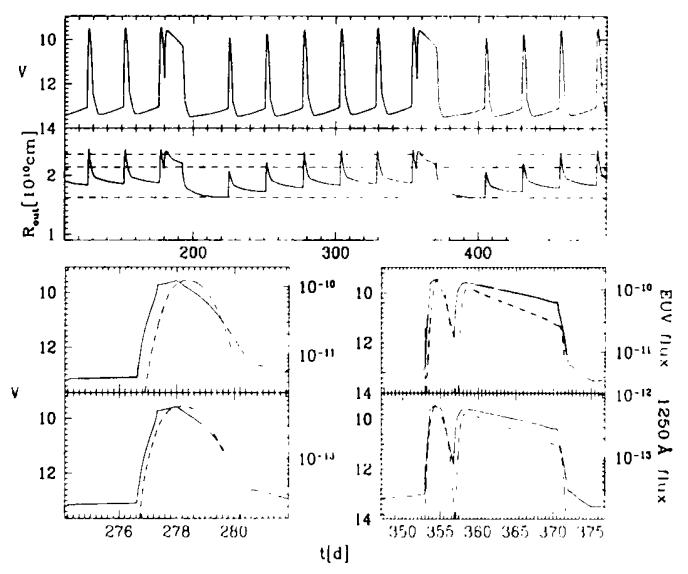

Fig. 2. Predictions of the EMIT for VIVHydri: optical light curve (top), the evolution of the outer radius (middle), and multi-wavelength snapshots (bottom). The dashed horizontal lines indicate the tidal truncation radius, the 3:1 resonance radius, and the radius at which the disc is assumed to become circular within the TTI. A superoutburst is triggered when enhanced mass transfer is causing a heating front overwhelming the precursor cooiing front (bottom, right). 